\title{
Estimation of resting blood pressure using facial thermal images by separating acute stress variations
}

\author{
Yuki Iwashita $^{1} \cdot$ Kent Nagumo $^{1} \cdot$ Kosuke Oiwa $^{1} \cdot$ Akio Nozawa $^{1}$
}

Received: 15 April 2021 / Accepted: 4 October 2021 / Published online: 11 October 2021

(C) The Author(s) 2021

\begin{abstract}
The increasing number of people with hypertension worldwide has become a matter of grave concern. Blood pressure monitoring using a non-contact measurement technique is expected to detect and control this medical condition. Previous studies have estimated blood pressure variations following an acute stress response based on facial thermal images obtained from infrared thermography devices. However, a non-contact resting blood pressure estimation method is required because blood pressure is generally measured in the resting state without inducing acute stress. Day-long blood pressure variations include short-term variations due to acute stress and long-term variations in circadian rhythms. The aim of this study is to estimate resting blood pressure from facial thermal images by separating and excluding short-term variations related to acute stress. To achieve this, short-term blood pressure variations components related to acute stress on facial thermal images were separated using independent component analysis. Resting blood pressure was estimated with the extracted independent components excluding the short-term components using multiple regression analysis. The results show that the proposed approach can accurately estimate resting blood pressure from facial thermal images, with a $9.90 \mathrm{mmHg}$ root mean square error. In addition, features related to resting blood pressure were represented in the nose, lip, and cheek regions.
\end{abstract}

Keywords Facial thermal image $\cdot$ Independent component analysis $\cdot$ Resting blood pressure

\section{Introduction}

The increasing number of people suffering from hypertension constitutes a social problem. Hypertension is a major risk factor for cardiovascular diseases and is associated with unhealthy lifestyle behaviors [1]. Daily blood pressure monitoring is important for prevention or early detection of these diseases. The conventional blood pressure measurement method applied pressure using a cuff attached to the finger or upper arm. This method has several disadvantages, such as personal discomfort and inability to continuously monitor blood pressure variations. A non-contact blood pressure

This work was presented in part at the 26th International Symposium on Artificial Life and Robotics (Online, January 2123, 2021).

Yuki Iwashita

yiwashita13@gmail.com

1 Aoyama Gakuin University, 5-10-1 Fuchinobe, Chuo-ku, Sagamihara, Kanagawa, Japan measurement technique can reduce these problems and facilitate daily blood pressure monitoring [2].

Visible and thermal images are physiological indices that reflected skin hemodynamic and non-contact measurable using a web camera or an infrared thermography device. Pulse waves can be measured from visible images based on the relationship between hue and blood flow fluctuation [3]. Skin temperature obtained from thermal images is related to skin blood flow, which is controlled by the sympathetic nervous system. The skin temperature variations depend on the heat conduction from the skin blood [4]. In particular, facial skin temperature shows a capacitive variation that reflects a dermovascular capacitance and the heat capacity of skin tissue [5]. This capacitive variation is spatially dependent on differences in blood vessel distribution and skin tissue structure in the facial skin. The techniques of non-contact blood pressure measurement have been developed using facial visible and thermal images.

In cardiovascular physiology, the Windkessel model is a hemodynamics model including blood pressure [6]. This model is an electric circuit model representing the cardiovascular system that consists of blood pressure as power, 
the peripheral vascular resistance as resistance, flexibility as capacitance, and blood flow as current. Blood pressure can be obtained from these parameters. Kato et al. estimated blood pressure based on the Windkessel model using electric current and charge from facial visible and thermal images, respectively [7]. This study was expanded to attempt a blood pressure estimation using only facial thermal images (FTIs).

In previous studies using FTIs, blood pressure was estimated when it was artificially elevated by acute stress response using deep learning algorithms [8] or independent component analysis (ICA) [9]. Blood pressure is generally measured in the resting state without inducing acute stress. However, to the best of our knowledge, a non-contact method for resting blood pressure estimation using FTIs has not been established.

Humans have a physiological response that varies over a 24-h cycle known as the circadian rhythm. Physiological indices vary throughout the day without acute stress because of the effects of the circadian rhythm $[10,11]$. Therefore, the day-long variation in physiological indices includes short-term variations related to acute stress and long-term variations related to circadian rhythms. Applying ICA to FTIs can extract independent components related to specific psychophysiological responses [12]. Ito et al. separated the components included in FTIs using ICA and extracted the core temperature or heart rate variable components related to circadian rhythms and acute stress [13]. It is expected that the short-term blood pressure variation components related to acute stress response can be separated and extracted from FTIs using the same method. In addition, resting blood pressure can be estimated using the independent components of FTIs, excluding the short-term variable component related to acute stress. This study aimed at estimating resting blood pressure from facial thermal images. In this first trial, the short-term blood pressure variations related to acute stress on FTIs were extracted and excluded using the method employed by Ito et al. FTIs and blood pressure were measured repeatedly throughout the day to evaluate variations related to circadian rhythm in each physiological index. For each experiment, a subject was provided with a task to induce acute stress to facilitate the separation of short-term components, such as artifacts. The task given was breath holding, which induced elevated blood pressure [14]. ICA was applied to each FTI obtained to extract the independent components. Correlation analysis was performed to extract short-term components related to acute stress. Multiple regression analysis was conducted to construct a model of resting blood pressure estimation using independent components excluding short-term components.

\section{Independent component analysis}

ICA is an algorithm that extracts a set of independent components from a set of random variables or signals $[15,16]$. In its simplest form, $m$ scalar random variables $x_{1}, \ldots x_{m}$ were observed. It is assumed that linear combinations of $n$ independent components exist, denoted by $s_{1}, \ldots s_{n}$. In this study, the observed variables $x_{j}$ and component variables $s_{i}$ were arranged as vectors $\mathbf{X}=\mathbf{x}_{j}(t)^{T}(j=1,2, \ldots, m) ; \mathbf{x}_{j}(t)$ have zero means, and $\mathbf{S}=\mathbf{s}_{i}(t)^{T}(i=1,2, \ldots, n)$. The linear relation is expressed as:

$\mathbf{X}=\mathbf{A S}=[\boldsymbol{a}(1), \boldsymbol{a}(2) \ldots, \boldsymbol{a}(t)]^{\mathrm{T}}\left[\boldsymbol{s}_{1}, \boldsymbol{s}_{2}, \ldots, \boldsymbol{s}_{n}\right]$

where $\mathbf{A}$, called the weighting matrix, is an unknown $t \times n$ matrix of the full column rank. $\mathbf{X}$ denotes the observed signal. The independent components are assumed to be mutually statistically independent and have zero means. ICA was used to extract the weighting matrix, $\mathbf{A}$ and independent components, $\mathbf{s}_{i}(m)$ from the observed signal alone.

\section{Experiment}

\subsection{Experimental system}

Figure 1 shows the experimental system. The physiological indices were FTI and mean arterial pressure (MAP). The experimental system comprised an infrared thermography device (A35 Series; FLIR Co.) and a non-invasive continuous blood pressure monitor (CNAP Monitor HD, CNSystems Co.). The infrared thermography device was set up approximately $1.0 \mathrm{~m}$ in front of the subject. The thermal images were captured at a sampling frequency of $1 \mathrm{~Hz}$. The size of the thermal image was $320 \times 256$ pixels. The temperature resolution was less than $0.05{ }^{\circ} \mathrm{C}$, and the infrared emissivity of the skin was set to $\epsilon=0.98$. MAP was measured by attaching a blood pressure cuff to the left finger and

Infrared thermography device

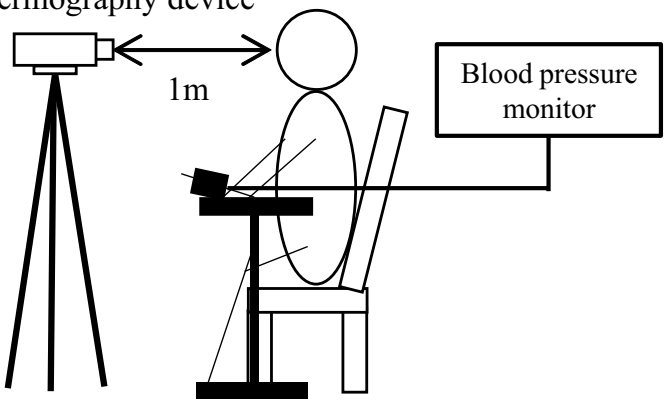

Fig. 1 Experimental system 


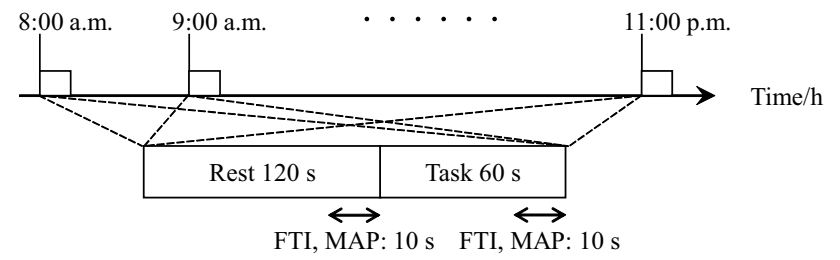

Fig. 2 Experimental protocol

upper arm with a non-invasive continuous blood pressure monitor at a $10 \mathrm{~Hz}$ sampling frequency.

\subsection{Procedure and condition}

The subject of this study was a healthy adult man aged 22 years. The subject was fully informed of the experimental procedure and the purpose of the study prior to participation. The study obtained official approval from the Life Science Committee of the College of Science and Engineering, Aoyama Gakuin University (Approval number:H17-M13-3). The temperature in the experimental room was set to $21.0 \pm 0.6^{\circ} \mathrm{C}$. Figure 2 shows the experimental protocol. The experiments were conducted every hour from 8:00 a.m. to 11:00 p.m. daily. The same experimental protocol was conducted for 3 days to confirm repeatability. To acclimatize to the temperature of the experiment room, the subject entered $15 \mathrm{~min}$ before the experiment began. To control the physiological responses to eating, the subject was only allowed three meals: breakfast at 8:00 a.m., lunch at 1:00 p.m., and dinner at 7:00 p.m. sleep time was required to be at least six hours. These restrictions were placed on eating and sleeping times to prevent changes in the circadian rhythm of physiological functions. The experiment consisted of a resting state segment (Rest) and an induced acute-stress physiological response segment (Task). In the Rest segment, subject was asked to rest with eyes closed for $120 \mathrm{~s}$. In the Task segment, subject was asked to hold breath with eyes closed for $60 \mathrm{~s}$ to induce acute stress. FTIs and MAP were measured continuously during the Rest and Task, and the last $10 \mathrm{~s}$ of each segment were used for analysis.

\section{Indices for analysis}

The analysis method comprised five procedures: (1) statistical evaluation, (2) extraction of FTIs, (3) extraction of independent components, (4) correlation analysis, and (5) multiple regression analysis. The details of each procedure are described in this section.

\subsection{Statistical evaluation}

Statistical evaluation was conducted to evaluate the physiological states. A two-factor analysis of variance (ANOVA) was used to measure MAP variations to assess the differences statistically between the two conditions, Rest and Task, and time lapse. A mean value of MAP for $10 \mathrm{~s}$ obtained from each experiment was used for the analysis.

\subsection{Extraction of facial thermal images (FTIs)}

FTIs were extracted from thermal images obtained in the experiment. A face detection algorithm based on a single shot multibox detector (SSD) and active appearance models (AAM) was used for FTIs extraction from thermal images $[17,18]$. An $82 \times 65$ pixel FTI was cropped from each thermal image based on the face detection algorithm, and the background or subject's hair was removed. Figure 3 shows an example of FTI. Each FTI was normalized to subtract blackbody temperature and mosaicked to remove temperature fluctuations caused by imperceptible facial movements.

\subsection{Extraction of independent components}

The observed signal was created from the extracted FTIs, and independent components were extracted based on ICA. The mean FTI was acquired from the FTIs measured for $10 \mathrm{~s}$ at time $t$ and expanded to a one-dimensional vector, represented as:

$\boldsymbol{x}(t)=\left[x_{1}(t), x_{2}(t), \ldots, x_{a \times b}(t)\right]$

where $x_{a \times b}(t)$ represents the pixel $a \times b$ value of the mean FTI at time $t . \mathbf{x}(t)$ are normalized to zero means. The mean FTI vectors for $t$ hours, which were extracted using a similar technique, were stored in a matrix:

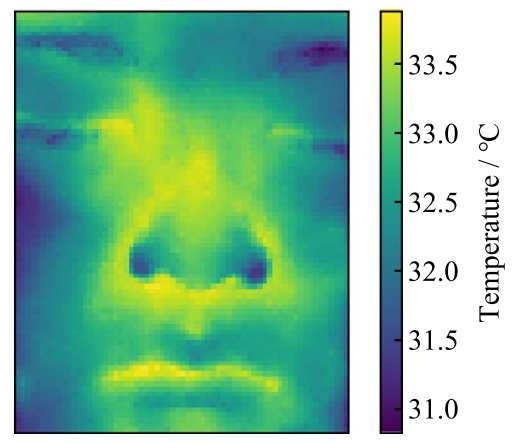

Fig. 3 Example of a FTI 
$\mathbf{X}_{\mathrm{FTI}}=[\boldsymbol{x}(1), \boldsymbol{x}(2), \ldots, \boldsymbol{x}(t)]^{\mathrm{T}}=\left[\begin{array}{ccc}x_{1,1} & \ldots & x_{1, a \times b} \\ \vdots & \ddots & \vdots \\ x_{t, 1} & \ldots & x_{t, a \times b}\end{array}\right]$

ICA applied to $\mathbf{X}_{\mathrm{FTI}}$. The weighting matrix $\mathbf{A}$ and independent components $\mathbf{S}$ were obtained using Eq. (1) as follows:

$\mathbf{A}=[\boldsymbol{a}(1), \boldsymbol{a}(2), \ldots, \boldsymbol{a}(t)]^{\mathrm{T}}=\left[\begin{array}{ccc}a_{1,1} & \ldots & a_{1, n} \\ \vdots & \ddots & \vdots \\ a_{t, 1} & \ldots & a_{t, n}\end{array}\right]$

$\mathbf{S}=\left[\boldsymbol{s}_{1}, \boldsymbol{s}_{2}, \ldots, \boldsymbol{s}_{n}\right]^{\mathrm{T}}=\left[\begin{array}{ccc}s_{1,1} & \ldots & s_{1, a \times b} \\ \vdots & \ddots & \vdots \\ s_{n, 1} & \ldots & s_{n, a \times b}\end{array}\right]$

The independent components $\mathbf{S}$ were represented the feature map of the face. The weighting variations $\mathbf{A}$ indicated time series variation. The fastICA algorithm [19-21], which outperforms the majority of commonly used ICA algorithms in terms of convergence speed, was used. The number of independent components was determined to be 13 based on negentropy $[22,23]$. The observed signal for applying ICA was all FTIs for 3 days. At time $t$, the FTIs for Rest and Task were included. This observed signal was defined as $\mathbf{X}_{\mathrm{ALL}}$. The extracted independent components and weighting variations from $\mathbf{X}_{\mathrm{ALL}}$ were defined as $\mathbf{S}_{\mathrm{ALL}}$ and $\mathbf{A}_{\mathrm{ALL}}$, respectively.

\subsection{Correlation analysis}

It is expected that $\mathbf{S}_{\mathrm{ALL}}$ includes long-term variable components on Rest or Task and short-term variable components due to acute stress. Correlation analysis was performed to separate short-term variable components in $\mathbf{S}_{\mathrm{ALL}}$. As shown in Table 1, FTIs were considered as observed signals, and the extracted independent components and weighting variations from each FTI were defined for correlation analysis. In Table 1, $\mathbf{X}_{\text {Rest }}$ and $\mathbf{X}_{\text {Task }}$ were observed signals created from FTIs during the Rest or Task, respectively. $\mathbf{S}_{\text {Rest }}, \mathbf{S}_{\text {Task }}, \mathbf{A}_{\text {Rest }}$, and $\mathbf{A}_{\text {Task }}$ are the independent components and weighting

Table 1 Defined observed signals, independent components, and weighting variations

\begin{tabular}{llcl}
\hline FTIs & Observed signal & $\begin{array}{l}\text { Independent } \\
\text { component }\end{array}$ & $\begin{array}{l}\text { Weight- } \\
\text { ing vari- } \\
\text { ation }\end{array}$ \\
\hline Rest in day $m$ & $\mathbf{X}_{\text {Rest } m}$ & $\mathbf{S}_{\text {Rest } m}$ & $\mathbf{A}_{\text {Rest } m}$ \\
Task in day $m$ & $\mathbf{X}_{\text {Task } m}$ & $\mathbf{S}_{\text {Task } m}$ & $\mathbf{A}_{\text {Task } m}$ \\
Rest for 3 days & $\mathbf{X}_{\text {Rest1-3 }}=\left[\mathbf{X}_{\text {Rest1 }}, \mathbf{X}_{\text {Rest2 }}, \mathbf{X}_{\text {Rest3 }} \mathbf{S}_{\text {est1-3 }}\right.$ & $\mathbf{A}_{\text {Rest1-3 }}$ \\
Task for 3 days & $\mathbf{X}_{\text {Task1-3 }}=\left[\mathbf{X}_{\text {Task1 } 1}, \mathbf{X}_{\text {Task2 } 2}, \mathbf{X}_{\text {Task } 39\} \text { k1-3 }}\right.$ & $\mathbf{A}_{\text {task1-3 }}$ \\
\hline
\end{tabular}

variations created from each observed signal. The number of independent components of $\mathbf{S}_{\text {Rest }}$ and $\mathbf{S}_{\text {Task }}$ was set to 13, similar to $\mathbf{S}_{\mathrm{ALL}}$. The correlation coefficients between $s_{n}(n=1,2, \ldots, 13)$ in $\mathbf{S}_{\mathrm{ALL}}$ and 13 independent components included in $\mathbf{S}_{\text {Rest }}$ or $\mathbf{S}_{\text {Task }}$ in Table 1 were calculated. If the maximum absolute value of the 13 correlation coefficients obtained was over $0.8, s_{n}$ is related to long-term variation of the Rest or Task. By contrast, if $\boldsymbol{s}_{n}$ in $\mathbf{S}_{\mathrm{ALL}}$ was not correlated with either $\mathbf{S}_{\text {Rest }}$ or $\mathbf{S}_{\text {Task }}$, then $\boldsymbol{s}_{n}$ was related to the short-term variation.

\subsection{Multiple regression analysis}

Multiple regression analysis was performed to construct the blood pressure estimation model from independent components, excluding the short-term component. Multiple regression analysis is represented as:

$y=\sum_{k=1} \beta_{k} x_{k}+$ Cnst.

where $\beta_{k}$ and Cnst. represent the partial regression coefficients and the constant term, respectively. Only MAP at rest was used for the blood pressure estimation model because this study aimed to estimate resting blood pressure. The dependent variable, $y$, was the mean MAP over $10 \mathrm{~s}$ obtained in each experiment at Rest for 3 days. The explanatory variables, $x_{k}$, were weighting variations $\mathbf{A}_{\mathrm{ALL}}$ excluding the independent components related to short-term variation identified in the correlation analysis. The explanatory variables were optimized using a stepwise procedure based on Akaike's information criterion (AIC) [24].

\section{Results and discussion}

\subsection{Independent component analysis to FTls}

Figure 4 shows the measured MAP variations in a subject over 3 days. The vertical axis, horizontal axis, and error bars indicate the mean value of MAP, time for 3 days, and standard error, respectively. The solid and dashed lines indicate the MAP in the Rest and Task segments, respectively. The ANOVA shows a significant main effect for conditions $(p<0.05)$ and time lapse $(p<0.001)$, and there was no interaction. The significant main effect for conditions was caused by blood pressure variation of the cardiac-dominant pattern induced by the task. On the other hand, it has been reported that blood pressure fluctuates based on circadian rhythms [25]. The significant main effect of time lapse is believed be caused by the circadian rhythm.

Figure 5 shows the result of $\mathbf{S}_{\mathrm{ALL}}$ and the corresponding $\mathbf{A}_{\mathrm{ALL}}$. In $\mathbf{S}_{\mathrm{ALL}}$, red and blue colors signify strong features, 


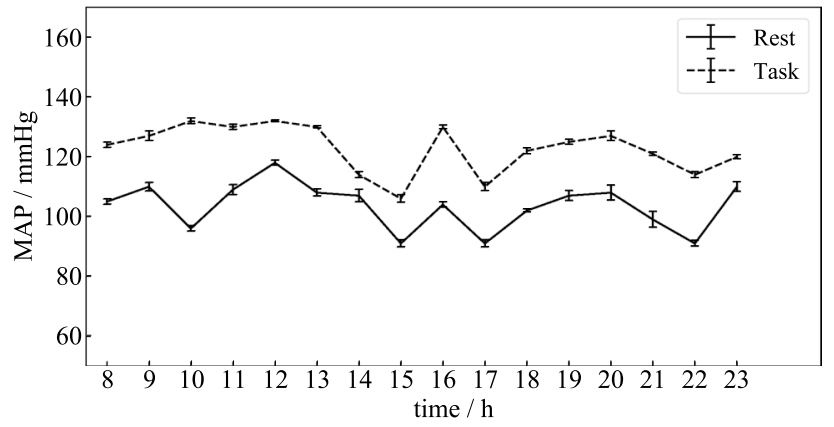

(a) Day 1

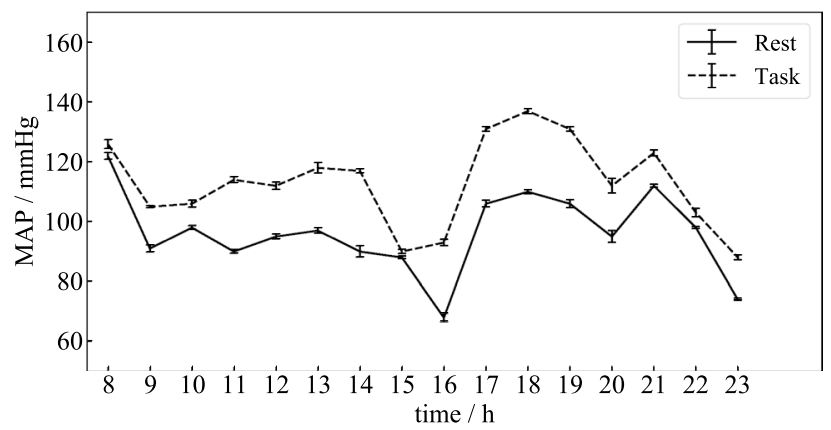

(b) Day 2

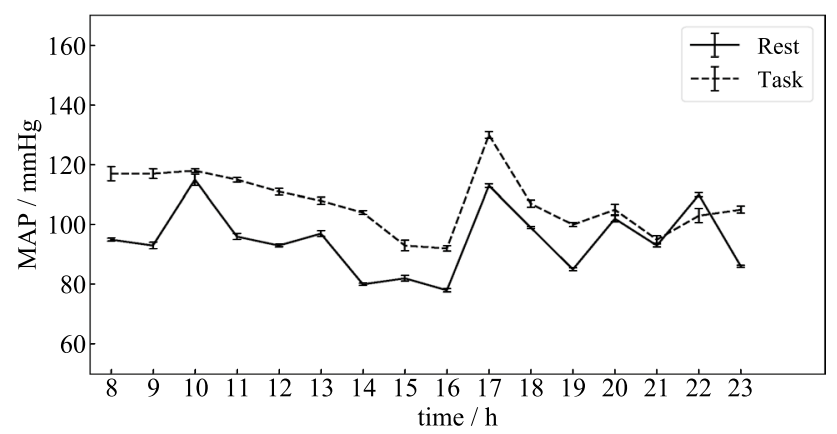

(c) Day 3

Fig. 4 Measured MAP variations. Error bars indicate the standard error

and white colors signify weak features. In $\mathbf{A}_{\mathrm{ALL}}$, the vertical and horizontal axes indicate the weighting values and the time for 3 days, respectively. Each day is divided by vertical lines.

The results of correlation analysis are shown in Table 2. If the maximum absolute value of thirteen correlation coefficients $(|R|)$ in is more than 0.8 , each cell in Table 2 is denoted by "O" and the others by "-". The $s_{12}$ and $s_{13}$ in $\mathbf{S}_{\mathrm{ALL}}$ were related to $\mathbf{S}_{\text {Task1-3 }}$ and $\mathbf{S}_{\text {Rest1-3 }}$, respectively, as shown in Table 2. These components are expected to be long-term components related to circadian rhythms and represent rhythms that are reproducible for three days. The $\boldsymbol{s}_{3}$ in $\mathbf{S}_{\mathrm{ALL}}$ was not correlated with the other observed signals, as shown in Table 2. The corresponding weighting variation
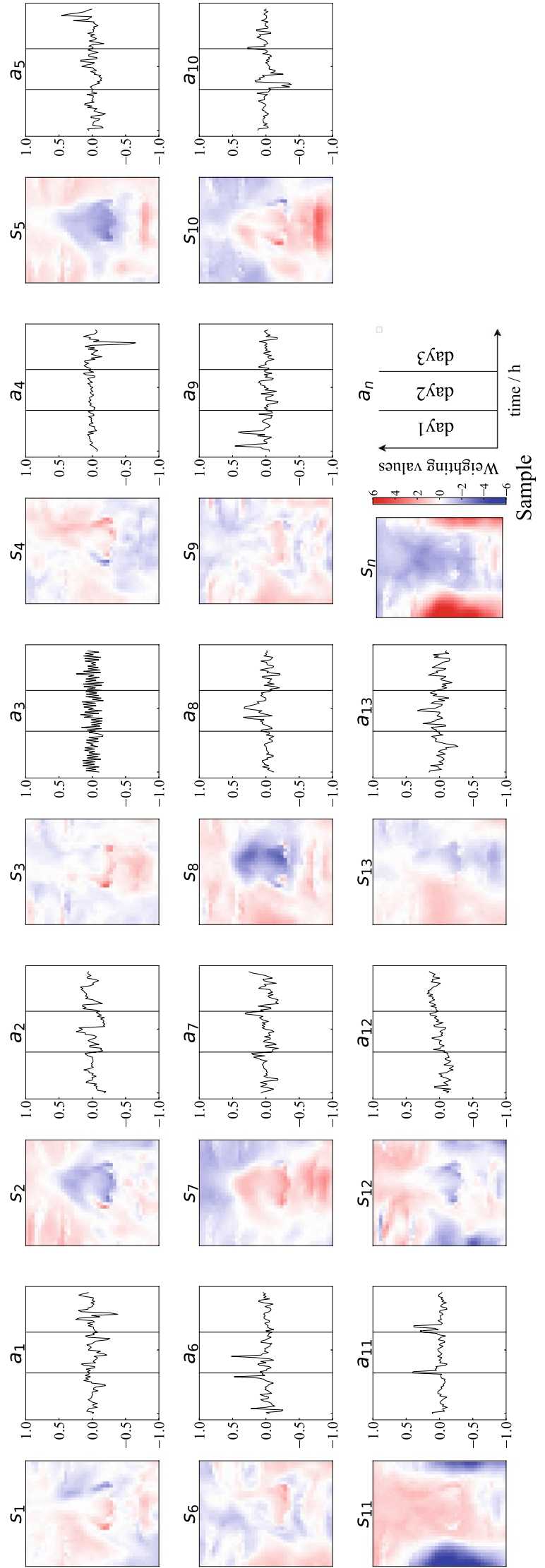
$\boldsymbol{a}_{3}$ qualitatively shows a time series of cyclic variation compared to other weighting variations, as shown in Fig. 5. ICA was applied to the FTIs dataset with Rest and Task arranged alternately because it assumed that the blood pressure variations were represented on the FTIs. It is considered that $\boldsymbol{a}_{3}$ was affected by blood pressure variations by acute stress. Therefore, $\boldsymbol{s}_{3}$ corresponding to $\boldsymbol{a}_{3}$ is expected to be a shortterm blood pressure variable component related to acute stress. It could be shown that a short-term blood pressure variable component related to acute stress could be separated and extracted from FTIs.

\subsection{Construction of model for resting blood pressure estimation}

The explanatory variables were $\mathbf{A}_{\mathrm{ALL}}$, excluding $\boldsymbol{a}_{3}$, which is the short-term variation component related to acute stress in the multiple regression analysis. Table 3 shows the results of multiple regression analysis. The shaded cells indicate excluded independent components, and the hyphen indicates the variables not selected as explanatory variables. Figure 6 shows the time variation of measured and estimated MAP. The vertical axis indicates the value of MAP, and the horizontal axis indicates time. The solid and dashed lines indicate the measured and estimated MAP, respectively. The coefficient of determination was 0.253 , and the root mean square error was $9.90 \mathrm{mmHg} . \boldsymbol{a}_{4}, \boldsymbol{a}_{7}, \boldsymbol{a}_{11}$, and $\boldsymbol{a}_{13}$ were selected as explanatory variables for the resting blood
Table 2 Correlation between $\mathbf{S}_{\mathrm{ALL}}$ and other independent components

Table 3 Results of multiple regression analysis

\begin{tabular}{|c|c|c|c|c|c|c|c|c|c|c|c|c|c|}
\hline & \multicolumn{13}{|c|}{ Number of $\mathbf{S}_{\mathrm{ALL}}$} \\
\hline & $s_{1}$ & $s_{2}$ & $s_{3}$ & $s_{4}$ & $s_{5}$ & $s_{6}$ & $s_{7}$ & $s_{8}$ & $s_{9}$ & $s_{10}$ & $s_{11}$ & $s_{12}$ & $s_{13}$ \\
\hline \multicolumn{14}{|l|}{ Rest } \\
\hline $\mathbf{S}_{\text {Rest1 }}$ & - & - & - & - & - & - & 0 & - & 0 & - & - & - & - \\
\hline $\mathbf{S}_{\text {Rest2 }}$ & - & - & - & - & - & - & 0 & 0 & - & O & 0 & - & - \\
\hline $\mathbf{S}_{\text {Rest3 }}$ & 0 & - & - & 0 & - & - & 0 & - & - & - & O & - & - \\
\hline $\mathbf{S}_{\text {Rest1-3 }}$ & - & - & - & 0 & O & - & O & 0 & 0 & O & O & - & O \\
\hline \multicolumn{14}{|l|}{ Task } \\
\hline $\mathbf{S}_{\text {Task1 }}$ & - & - & - & - & - & O & 0 & - & - & - & - & - & - \\
\hline $\mathbf{S}_{\text {Task2 }}$ & - & 0 & - & - & - & 0 & - & - & 0 & - & 0 & - & - \\
\hline $\mathbf{S}_{\text {Task3 }}$ & - & - & - & O & O & - & - & - & -- & - & 0 & - & - \\
\hline $\mathbf{S}_{\text {Task1-3 }}$ & - & - & - & 0 & 0 & 0 & 0 & 0 & 0 & 0 & 0 & 0 & - \\
\hline
\end{tabular}

"O" represents that correlation coefficients $(R)$ are greater than the threshold value $(|R|>0.8)$, and the others are represented by “-”"

\begin{tabular}{|c|c|c|c|c|c|c|c|c|c|c|c|c|c|c|c|}
\hline \multirow[t]{2}{*}{ Cnst } & \multicolumn{13}{|c|}{ Standard partial regression coefficients corresponding to $\beta_{k}$ to $\mathbf{A}_{\mathrm{ALL}}$} & \multirow[t]{2}{*}{$R^{2}$} & \multirow{2}{*}{$\begin{array}{l}\text { RMSE } \\
/ \mathrm{mmHg}\end{array}$} \\
\hline & $\beta_{1}$ & $\beta_{2}$ & $\beta_{3}$ & $\beta_{4}$ & $\beta_{5}$ & $\beta_{6}$ & $\beta_{7}$ & $\beta_{8}$ & $\beta_{9}$ & $\beta_{10}$ & $\beta_{11}$ & $\beta_{12}$ & $\beta_{13}$ & & \\
\hline 98.2 & - & - & - & 2.33 & - & - & -2.16 & - & - & - & 3.30 & - & -3.49 & 0.253 & 9.90 \\
\hline
\end{tabular}

Bold cells indicate the excluded independent components. The hyphen indicates the variables not selected as explanatory variables

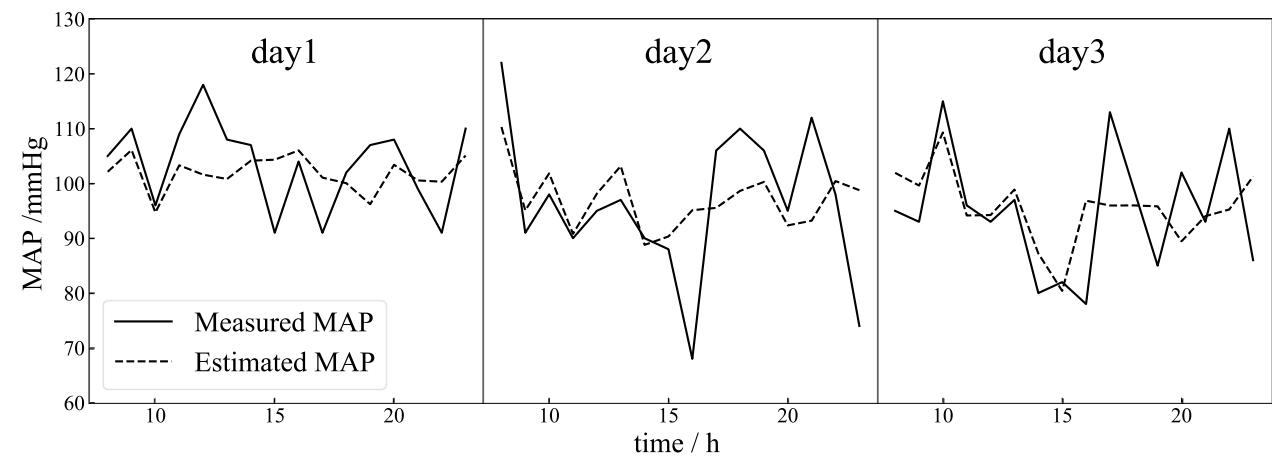


pressure estimation model. Therefore, $\boldsymbol{s}_{4}, \boldsymbol{s}_{7}, \boldsymbol{s}_{11}$, and $\boldsymbol{s}_{13}$ were independent components related to the resting blood pressure variation. Additionally, $\boldsymbol{s}_{13}$ corresponding to $\boldsymbol{a}_{13}$ was a long-term component related to circadian rhythm, as mentioned in Sect. 5.1. $\boldsymbol{s}_{13}$ was expected to represent the blood pressure variable components related to the main circadian rhythm. The coloration in the nose in $s_{4}$, lip in $s_{7}$ and $s_{13}$, and cheek in $s_{11}$ regions were intensified as shown in Fig. 5, and these regions were suggested to be related to resting blood pressure. In a previous study, the nose region was extracted as feature quantities related to short-term blood pressure variations based on acute stress [26]. The nasal and lip region is well known as the peripheral region reflecting the blood flow variation [27]. Therefore, it is considered that regions reflecting blood flow fluctuations are more likely to be used to estimate resting blood pressure.

\section{Conclusion}

The objective of this study was to estimate resting blood pressure by separating acute stress blood pressure variations using FTIs. ICA was applied to the FTIs to extract features related to acute stress blood pressure variations. From the extracted independent components, a correlation analysis was used to separate and extract the short-term variable components related to acute stress. Multiple regression analysis was performed to estimate resting blood pressure using independent components, excluding short-term variable components. As a result, resting blood pressure was estimated from FTIs with an accuracy of $9.90 \mathrm{mmHg}$ root mean square error between measured and estimated MAP. The features related to resting blood pressure variation were represented in the nose, lip, and cheek regions. The contribution of this study is the separation and extraction of short-term components related to acute stress blood pressure variations, and resting blood pressure could be estimated with an accuracy of $9.90 \mathrm{mmHg}$.

This study has a limitation owing to its lack of generality, because only one subject was involved. In future studies, it is necessary to test the generality of resting blood pressure estimation using FTIs by increasing the number of subjects and comparing the obtained results. Additionally, the components of FTIs need to be evaluated while considering interday differences in blood pressure, because a difference was observed in blood pressure variations on day 1,2 , and 3 .

Open Access This article is licensed under a Creative Commons Attribution 4.0 International License, which permits use, sharing, adaptation, distribution and reproduction in any medium or format, as long as you give appropriate credit to the original author(s) and the source, provide a link to the Creative Commons licence, and indicate if changes were made. The images or other third party material in this article are included in the article's Creative Commons licence, unless indicated otherwise in a credit line to the material. If material is not included in the article's Creative Commons licence and your intended use is not permitted by statutory regulation or exceeds the permitted use, you will need to obtain permission directly from the copyright holder. To view a copy of this licence, visit http://creativecommons.org/licenses/by/4.0/.

\section{References}

1. Forouzanfar MH, Liu P, Roth GA, Ng M, Biryukov S, Marczak L, Murray CJ et al (2017) Global burden of hypertension and systolic blood pressure of at least 110-115 mm Hg, 1990-2015. J Am Med Assoc 317(2):165-182

2. Ding XR, Zhao N, Yang GZ, Pettigrew RI, Lo B, Miao F, Zhang YT et al (2016) Continuous blood pressure measurement from invasive to unobtrusive: celebration of 200th birth anniversary of Carl Ludwig. IEEE J Biomed Health Inform 20(6):1455-1465

3. Jonathan E, Leahy M (2010) Investigating a smartphone imaging unit for photoplethysmography. Physiol Meas 31(11):N79-83

4. Haga T, Ibe A, Aso Y, Ishizawa M, Miyajima M, Takeda K (2012) Development of methodology for the estimation of skin blood perfusion by applying inverse analysis of skin model. Trans Jpn Soc Med Biol Eng 50(4):317-328

5. Mizuno T, Nomura S, Nozawa A, Asano H, Ide H (2010) Evaluation of the effect of intermittent mental workload by nasal skin temperature. IEICE Trans Inf Syst J93-D(4):535-543

6. Wesseling H, Jansen JRC, Settels JJ, Schreuder JJ (1993) Computation of aortic flow from pressure in humans using a nonlinear, three-element model. J Appl Physiol 74(5):2566-2573

7. Kato Y, Nagumo K, Bando S, Oiwa K, Nozawa A (2019) Electric circuit model and thermo-hue hemodynamic analysis for noncontact blood pressure measurement. IEEJ Trans Electron Inf Syst 140(1):122-123

8. Oiwa K, Nozawa A (2019) Feature extraction of blood pressure from facial skin temperature distribution using deep learning. IEEJ Trans Electron Inf Syst 139(7):759-765

9. Nakane N, Oiwa K, Nozawa A (2020) Construction of a general model for estimating blood pressure using independent components of facial skin temperature in consideration of the mechanism of variation. In: 2020 IEEE 18th World symposium on applied machine intelligence and informatics (SAMI), pp 145-150

10. Massin MM, Maeyns K, Withofs N, Ravet F et al (2000) Circadian rhythm of heart rate and heart rate variability. Arch Dis Child 83(2):179-182

11. Weinert D, Waterhouse J (2007) Circadian rhythm of core temperature: effects of physical activity and aging. Physiol Behav 90(2-3):246-256

12. Okamoto R, Bando S, Nozawa A (2016) Blind signal processing of facial thermal images based on independent component analysis. IEEJ Trans Electron Inf Syst 136(8):1142-1148

13. Ito H, Bando S, Oiwa K, Nozawa A (2018) Evaluation of variations in autonomic nervous system activity during the day based on facial thermal images using independent component analysis. IEEJ Trans Electron Inf Syst 138(7):1-10

14. Porth CJ, Bamrah VS, Tristani FE et al (1984) The Valsalva maneuver: mechanisms and clinical implications. Heart Lung 13(5):507-518

15. Comon P (1994) Independent component analysis, a new concept? Signal Process 36(3):287-314

16. James CJ, Hesse CW (2004) Independent component analysis for biomedical signals. Physiol Meas 26(1):R15

17. Kopaczka M, Blanik N, Czaplik M, Hochhausen N, Paul M, Pereira C, Blazek V, Leonhardt S, Merhof D (2015) A thermal 
infrared face database and active appearance model based face detection in a system for pain assessment in sedated patients. In: Proceedings of the 11th German-Russian conference on Biomedical Engineering, p 3336

18. Nagumo K, Kobayashi T, Oiwa K, Nozawa A (2021) Face alignment in thermal infrared images using cascaded shape regression. Int J Environ Res Public Health 18(4):1776

19. Hyvärinen A, Oja E (1997) A fast fixed-point algorithm for independent component analysis. Neural Comput 9(7):1483-1492

20. Hyvärinen A (1999) Fast and robust fixed-point algorithms for independent component analysis. IEEE Trans Neural Netw 10(3):626-634

21. Oja E, Zhijian Y (2006) The FastICA algorithm revisited: convergence analysis. IEEE Trans Neural Netw 17(6):1370-1381

22. Sasaoka H, Kirino S (2018) Independent component analysis and its application to blind multi-input multi-output systems. Harris Sci Rev Doshisya Univ 59(3):135-145
23. Hyvärinen A, Karhunen J, Oja E (2001) Independent component analysis. Wiley, Hoboken

24. Yamashita T, Yamashita K, Kamimura R (2007) A stepwise AIC method for variable selection in linear regression. Commun Stat Theory Methods 36(13):2395-2403

25. Biaggioni I (2008) Circadian clocks, autonomic rhythms, blood pressure dipping. Hypertension 52:797-798

26. Nakane N, Oiwa K, Nozawa A (2020) Relationship between mechanisms of blood pressure change and facial skin temperature distribution. Artif Life Robot 25(1):48-58

27. Bergersen TK (1993) A search for arteriovenous anastomoses in human skin using ultrasound Doppler. Acta Physiol Scand 147(2):195-201 